\title{
Volume of the effect compartment in simulations of neuromuscular block
}

\author{
Vladimir Nigrovic*1, Johannes H Proost ${ }^{2}$, Anton Amann ${ }^{3}$ and \\ Shashi B Bhatt ${ }^{1}$
}

Address: ${ }^{1}$ Department of Anesthesiology, Medical University of Ohio, Toledo, OH, USA, ${ }^{2}$ Research Group for Experimental Anesthesiology and Clinical Pharmacology, University Hospital Groningen, Groningen, The Netherlands and ${ }^{3}$ Department of Anesthesiology and Critical Care Medicine, Leopold-Franzens University, Innsbruck, Austria, and Department of Environmental Sciences, The Swiss Federal Institute of Technology, Zürich, Switzerland

Email: Vladimir Nigrovic* - vnigrovic@meduohio.edu; Johannes H Proost - j.h.proost@rug.nl; Anton Amann - Anton.Amann@uibk.ac.at; Shashi B Bhatt - sbhatt@meduohio.edu

* Corresponding author

Published: 03 October 2005

Theoretical Biology and Medical Modelling 2005, 2:41 doi:10.1 186/|742-4682-2-4|

This article is available from: http://www.tbiomed.com/content/2/l/4I

(C) 2005 Nigrovic et al; licensee BioMed Central Ltd.

This is an Open Access article distributed under the terms of the Creative Commons Attribution License (http://creativecommons.org/licenses/by/2.0), which permits unrestricted use, distribution, and reproduction in any medium, provided the original work is properly cited.
Received: 02 September 2005

Accepted: 03 October 2005

\begin{abstract}
Background: The study examines the role of the volume of the effect compartment in simulations of neuromuscular block (NMB) produced by nondepolarizing muscle relaxants.

Methods: The molar amount of the postsynaptic receptors at the motor end plates in muscle was assumed constant; the apparent receptor concentration in the effect compartment is the ratio of this amount and the volume arbitrarily assigned to the effect compartment. The muscle relaxants were postulated to diffuse between the central and the effect compartment and to bind to the postsynaptic receptors. NMB was calculated from the free concentration of the muscle relaxant in the effect compartment.
\end{abstract}

Results: The simulations suggest that the time profiles of NMB and the derived pharmacokinetic and pharmacodynamic variables are dependent on the apparent receptor concentration in the effect compartment. For small, but not for large, volumes, times to peak submaximal NMB are projected to depend on the magnitude of NMB and on the binding affinities.

Conclusion: An experimental design to estimate the volume of the effect compartment is suggested.

\section{Background}

In the majority of the pharmacokinetic-pharmacodynamic (PK-PD) models proposed to simulate neuromuscular block (NMB) [1-3], the volume of the effect compartment is postulated to be negligibly small or the compartment is postulated to contain a negligibly small amount of the muscle relaxant. The models simulate NMB based on the concentration of the muscle relaxant in this compartment using the equation of Hill. Binding of muscle relaxants to the postsynaptic receptors at the motor end plates is not considered. Because muscle relaxants produce NMB by binding to these receptors, consideration of the interaction of muscle relaxants with the receptors represents a more realistic approach and an advancement in simulations [4-6]. Donati and Meistelman [5] were the first to consider binding of muscle 
relaxants to the receptors. These investigators suggested that the receptor concentration in the effect compartment is $2.8 \cdot 10^{-7} \mathrm{M}$, but the volume of the effect compartment was assumed to be negligibly small. Given a fixed amount of postsynaptic receptors, a finite receptor concentration is not compatible with a negligibly small volume of the effect compartment.

We decided to examine the role of the volume of the effect compartment in a pharmacokinetic-pharmacodynamic model for NMB and were interested in answering the following questions: (1) Is it necessary to postulate a negligibly small amount of a muscle relaxant in the effect compartment? (2) Do the projections from simulations using a small or a large volume of the effect compartment differ? If so, what are the differences? (3) Can the simulations suggest an experimental design suitable to test whether the volume of the effect compartment is negligibly small or a large volume may be more appropriate?

\section{Methods}

\section{General approach}

(1) The amount of the postsynaptic receptors at the motor end plates in muscle, in terms of mol per kg body weight, was assumed constant and the receptors uniformly diluted in the effect compartment. (2) The plasma concentrations of a hypothetical muscle relaxant after administration of an intravenous bolus dose, defined by an arbitrary multiexponential equation, are labeled target concentrations. In the simulations, the target plasma concentrations fulfill the role of the experimentally determined plasma concentrations. (3) A PK-PD model was designed a priori to include an effect compartment of an assigned volume. The pharmacokinetic parameters in the model were defined by the postulate that the concentrations in the central compartment (compartment1) fit the target plasma concentrations. (4) The muscle relaxant diffuses from the central to the effect compartment. (5) Pharmacodynamic parameters were obtained from the postulate that peak neuromuscular block from a bolus ED50 dose occurs at 4.5 minutes after injection. The peak concentration of the muscle relaxant in the effect compartment at this moment corresponds to the IC50 concentration. (6) The relationship between NMB and the free concentrations of the muscle relaxant in the effect compartment is defined by the Hill equation.

\section{The target plasma concentrations}

Muscle relaxant D was postulated to display linear pharmacokinetics. The triexponential equation that defines the time course of the molar amounts of the muscle relaxant in plasma is given by (braces indicate molar amounts):

$$
\{D\}_{\text {plasma }}=\operatorname{dose} \cdot\left(N \cdot e^{-\lambda_{N} \cdot t}+O \cdot e^{-\lambda_{O} \cdot t}+P \cdot e^{-\lambda_{P} \cdot t}\right)
$$

Here, $\mathrm{N}, \mathrm{O}$, and $\mathrm{P}(\mathrm{P}=1-\mathrm{N}-\mathrm{O})$ are fractions of the dose that are eliminated from plasma with the first order rate constants $\lambda_{\mathrm{N}^{\prime}}, \lambda_{\mathrm{O}^{\prime}}$ and $\lambda_{\mathrm{p}}$, respectively. The dose is in $\mathrm{mol} \cdot \mathrm{kg}^{-1}$. Division of the equation by $\mathrm{V}_{\mathrm{C}^{\prime}}, \mathrm{V}_{\mathrm{C}}$ expressed in $\mathrm{L} \cdot \mathrm{kg}^{-1}$, converts the amounts in plasma to molar concentrations. $\mathrm{V}_{\mathrm{C}}$ represents the volume of the space into which the muscle relaxant is uniformly diluted at time $\mathrm{t}=0$, i.e., at the moment of bolus intravenous injection.

The values assigned to the parameters in the triexponential equation were based on the following postulates: For the hypothetical muscle relaxant $\mathrm{D}, \mathrm{V}_{\mathrm{C}}$ approximates the volume of plasma and $\mathrm{V}_{\mathrm{SS}}$, the volume of distribution at steady state, approximates the volume of the extracellular space. The dose that produces NMB50, i.e., ED50, is defined by the postulate that the concentration in plasma at $4.5 \mathrm{~min}$ after bolus intravenous injection is $[\mathrm{D}]_{\text {plasma }}=$ IC50. The definition of IC50 is provided below. The following values satisfy these requirements:

$$
\begin{aligned}
& \mathrm{N}=0.71 ; \mathrm{O}=0.192 ; \mathrm{P}=0.098 \\
& \lambda_{\mathrm{N}}=1.3 \mathrm{~min}^{-1} ; \lambda_{\mathrm{O}}=0.31 \mathrm{~min}^{-1} ; \lambda_{\mathrm{P}}=0.0231 \mathrm{~min}^{-1} \\
& \mathrm{~V}_{\mathrm{C}}=0.044 \mathrm{~L} \cdot \mathrm{kg}^{-1} \mathrm{~V}_{\mathrm{SS}}=0.28 \mathrm{~L} \cdot \mathrm{kg}^{-1}
\end{aligned}
$$

Compartmental interpretation of the triexponential decay of the plasma concentrations yields the following parameters for the standard 3-compartment pharmacokinetic model assuming a mammillary arrangement of the compartments and elimination only from compartment ${ }_{1}[7]$ :

$$
\begin{aligned}
& \mathrm{V}_{1}=\mathrm{V}_{\mathrm{C}}=0.044 \mathrm{~L} \cdot \mathrm{kg}^{-1} \mathrm{k}_{10}=0.1848 \mathrm{~min}^{-1} \\
& \mathrm{k}_{12}=0.3771 \mathrm{~min}^{-1} \mathrm{k}_{21}=0.5581 \mathrm{~min}^{-1} \\
& \mathrm{k}_{13}=0.4229 \mathrm{~min}^{-1} \mathrm{k}_{31}=0.0902 \mathrm{~min}^{-1}
\end{aligned}
$$

\section{Estimation of the receptor amount}

The molar amount of receptors per kg body weight was estimated based on the following assumptions: One hundred $\mathrm{g}$ of muscle is represented as a cube with side length of $4.64 \mathrm{~cm}$, i.e., specific density of muscle $\sim 1$. There is 430 $\mathrm{g}$ muscle per $\mathrm{kg}$ body weight. The muscle fibers are densely packed cylinders with the diameter of $50 \mu \mathrm{m}$ and the length of $4.64 \mathrm{~cm}$ ( 928 rows $\times 928$ columns of fibers in a cross section perpendicular to the length of the fibers). Each muscle fiber has one motor end plate with $2.1 \cdot 10^{7}$ receptors at each end plate $[8,9]$. 


\section{The PK-PD Model}

The pharmacokinetic model consists of four compartments: the central (compartment ${ }_{1}$ ), two peripheral (compartment ${ }_{2}$ and compartment ${ }_{3}$ ), and the effect compartment in mammillary arrangement with elimination from the central compartment. The model is defined in terms of the amounts of the muscle relaxant present in each compartment and the amount eliminated from the body. Transport between the central and the effect compartment is defined as diffusion according to the concentration gradient of the free muscle relaxant in both compartments. As a result, at the moment when the free muscle relaxant attains the peak concentration in the effect compartment and there is no net transport between the compartments (steady state), the concentrations in the two compartments are equal. In the model, this constraint necessitates that the transport rate constant into the effect compartment, $\mathrm{k}_{1 e^{\prime}}$, be defined in terms of the transport rate constant from the effect to the central compartment, $\mathrm{k}_{\mathrm{e} 1}$. Hence, $\mathrm{k}_{1 e}=\left(\mathrm{V}_{\mathrm{e}} / \mathrm{V}_{1}\right) \cdot \mathrm{k}_{\mathrm{e} 1}$, where $\mathrm{V}_{\mathrm{e}}$ and $\mathrm{V}_{1}$ represent the volumes of the effect and the central compartments, respectively. The volume of the central compartment is known $\left(\mathrm{V}_{1} \approx \mathrm{V}_{\mathrm{C}}\right.$ in the triexponential function). The volume of the effect compartment was assigned different values. Hence, the amounts of $\mathrm{D}$ in the central and the effect compartments may be converted to concentrations. Compartment ${ }_{2}$ and compartment core $_{3}$ defined only in terms of the amounts present in them.

The amount of receptors in the effect compartment is constant and independent of the volume assigned to the effect compartment. A small assigned volume results in a high receptor concentration, while the concentration is low in the large effect compartment.
For an assigned volume of the effect compartment $\left(\mathrm{V}_{\mathrm{e}}\right)$, the pharmacokinetic parameters in the PK-PD model were estimated in a two-step procedure. In the first step, the parameter $k_{e 1}$ was obtained using the following constraints: dose $=$ ED50, the amounts in plasma as defined by the triexponential equation, and the maximal $\mathrm{NMB}=$ $50 \%$ attained at 4.5 min after administration of the muscle relaxant. In the second step, the parameters $\mathrm{V}_{1}, \mathrm{k}_{10}, \mathrm{k}_{12}$, $\mathrm{k}_{21}, \mathrm{k}_{13}$, and $\mathrm{k}_{31}$ were estimated using the following constraints: dose $=$ ED50 and $\mathrm{k}_{\mathrm{e} 1}$ fixed to the value obtained in the first step. The parameters were fitted by minimizing the sum of squared differences between the logarithms for the calculated concentrations in compartment ${ }_{1}$ and the target concentrations in plasma. The evaluations were carried out at 250 time points from $\mathrm{t}=0$ to $\mathrm{t}=25 \mathrm{~min}$ and at 50 points for $\mathrm{t}=25$ to $\mathrm{t}=50 \mathrm{~min}$ after administration. Goodness-of fit was expressed as the coefficient of variation ( $\mathrm{CV}$ in $\%{ }^{1}$ ) of the differences between the two time profiles.

Interaction between the muscle relaxant and the postsynaptic receptors was defined in terms of the association, $\mathrm{k}_{\mathrm{as}}$ ${ }_{\text {soc }}$, and dissociation, $\mathrm{k}_{\mathrm{dis}^{\prime}}$ rate constants. We assumed that each receptor possesses only a single binding site for the muscle relaxant. The ratio $\mathrm{k}_{\mathrm{dis}} / \mathrm{k}_{\mathrm{assoc}}$ defines the equilibrium dissociation constant, $\mathrm{K}_{\mathrm{D}}$. The inverse of $\mathrm{K}_{\mathrm{D}}$ defines the affinity of the receptors for the muscle relaxant.

The values of all the mentioned parameters are listed in Table 1 . The set of five ordinary differential equations defining the amounts of the muscle relaxant in the four compartments and the amount of the complex with the receptors in the effect compartment is presented in the Appendix.

Table I: Pharmacokinetic and pharmacodynamic parameters for the PK-PD model. The volume of the effect compartment $\left(V_{e}\right)$ was postulated to be either small (SMALL) or large (LARGE).

\begin{tabular}{|c|c|c|c|c|}
\hline Parameter & Unit & SMALL & & LARGE \\
\hline ED50 & $\mathrm{mol} \cdot \mathrm{kg}^{-1}$ & & $2.2325 \cdot 10^{-7}$ & \\
\hline$v_{1}$ & ${\mathrm{~L} \cdot \mathrm{kg}^{-1}}^{-1}$ & 0.0440 & & 0.0434 \\
\hline$k_{10}$ & $\min ^{-1}$ & 0.1847 & & 0.1795 \\
\hline$k_{12}$ & $\min ^{-1}$ & 0.3769 & & 0.3574 \\
\hline$k_{21}$ & $\min ^{-1}$ & 0.5587 & & 0.7663 \\
\hline$k_{13}$ & $\min ^{-1}$ & 0.4226 & & 0.1981 \\
\hline$k_{31}$ & $\min ^{-1}$ & 0.0909 & & 0.0581 \\
\hline $\mathrm{k}_{\text {assoc }}$ & $\mathrm{M}^{-1} \cdot \mathrm{min}^{-1}$ & & $2.4 \cdot 10^{10}$ & \\
\hline$\{R\}_{\text {total }}$ & $\mathrm{mol} \cdot \mathrm{kg}^{-1}$ & & $1.2921 \cdot 10^{-10}$ & \\
\hline $\mathrm{V}_{\mathrm{e}}$ & $\mathrm{L} \cdot \mathrm{kg}^{-1}$ & $4.4 \cdot 10^{-5}$ & & $9.23 \cdot 10^{-2}$ \\
\hline$k_{e l}$ & $\min ^{-1}$ & 0.6159 & & 0.1477 \\
\hline$[R]_{\text {total }}$ & $M$ & $2.9367 \cdot 10^{-6}$ & & $1.4 \cdot 10^{-9}$ \\
\hline Onset time & $\min$ & & 4.50 & \\
\hline
\end{tabular}




\section{Calculation of NMB}

The intravenous bolus dose of the muscle relaxant required to produce a half-maximal NMB, NMB50, is labeled ED50. We postulated that NMB50 is attained at $4.5 \mathrm{~min}$ after the bolus injection. The peak concentration of the free muscle relaxant in the effect compartment established by ED50 is IC50. At $4.5 \mathrm{~min}$ after injection, $[D]_{\text {plasma }}=$ peak $[D]_{e}=$ IC50. The fractional receptor occupancy by the muscle relaxant (Occ) at NMB50 is labeled Occ $_{\mathrm{NMB} 50}$ and assigned a value of 0.875 [10]. Because $\mathrm{K}_{\mathrm{D}}$ $=[\mathrm{D}]_{e} \cdot(1-\mathrm{Occ}) / \mathrm{Occ}$, and at NMB50 $[\mathrm{D}]_{e}=\mathrm{IC} 50$ and Occ $=$ Occ $_{\text {NMB50 }}=0.875$, it follows that IC50 $=7 \cdot \mathrm{K}_{\mathrm{D}}$.

Neuromuscular block (NMB) was calculated using the Hill equation, the free concentrations of the muscle relaxant in the effect compartment, $[\mathrm{D}]_{\mathrm{e}^{\prime}}$ and two parameters: $\gamma$ and IC50 $\left(\gamma=4\right.$ and IC50 $=7 \cdot \mathrm{K}_{\mathrm{D}^{\prime}}$ Eq 1 in Appendix $)$.

To describe quantitatively the simulated NMB as a function of doses used to establish the peak concentrations in the effect compartment, the values for NMB calculated from peak $[\mathrm{D}]_{e}$ were plotted as a function of doses of the muscle relaxant. A modified equation of Hill (Eq. 2, Appendix) was fitted to these points using the program TableCurve2D from SPSS, Chicago, IL, and the fitted estimates of the exponent $\gamma_{\mathrm{f}}$ and $\mathrm{ED} 50_{\mathrm{f}}$ are reported.

All calculations were performed independently using the programs MATHEMATICA (version 5.1) from Wolfram Research, Inc., Champaign, IL, MULTIFIT and PKPDFIT written by J.H. Proost, and MATLAB (version 6.1.0.450(R12.1)) from The Mathworks Inc., Natick, MA.

\section{Results}

The estimated total molar amount of receptors at the motor end plates in muscles is $\{\mathrm{R}\}_{\text {total }}=1.2921 \cdot 10^{-10}$ $\mathrm{mol} \cdot \mathrm{kg}^{-1}$. Receptor concentration in the effect compartment is the ratio of this amount and the volume assigned to the effect compartment.

\section{Simulations with a small or a large volume assigned to the effect compartment}

For the initial simulations, $\mathrm{V}_{\mathrm{e}}$ was assigned the value of $0.001 \cdot \mathrm{V}_{\mathrm{C}}$, i.e., $\mathrm{V}_{\mathrm{e}}=4.4 \cdot 10^{-5} \mathrm{~L} \cdot \mathrm{kg}^{-1}[11]$, for the small and $0.0923 \mathrm{~L} \cdot \mathrm{kg}^{-1}$ for the large effect compartment. The latter approximates the volume of the interstitial space in muscle. Receptor concentrations in the effect compartment were: $[R]_{\text {total }}=2.94 \cdot 10^{-6} \mathrm{M}$ and $1.4 \cdot 10^{-9} \mathrm{M}$ for the small and large volume, respectively. The hypothetical muscle relaxant $\mathrm{D}$ was assigned $\mathrm{K}_{\mathrm{D}}=1 \cdot 10^{-7} \mathrm{M}$. The assignment defined ED50 as ED50 $=2.23 \cdot 10^{-7} \mathrm{~mol} \cdot \mathrm{kg}^{-1}$. Optimal estimates of the pharmacokinetic parameters, including $\mathrm{k}_{\mathrm{e} 1}$, were obtained as described in the Methods section. The target amounts of the muscle relaxant in plasma and those estimated in compartment ${ }_{1}$ as well as the amounts in the small and the large effect compartments are graphically presented in the upper panel of Figure 1 . The three curves for the amounts in plasma overlap. The good fit of the amounts in compartment ${ }_{1}$ to the target amounts in plasma is evident from the small values of the coefficient of variation, $0.0007 \%$ for the small and $0.7 \%$ for the large volume of the effect compartment. The peak free amount of $\mathrm{D}$ in the small effect compartment constitutes a small fraction of ED50, $1.38 \cdot 10^{-4}$. On the other hand, the peak free amount of $\mathrm{D}$ in the large effect compartment accounts for a sizable fraction of ED50, 0.289 (upper panel in Figure 1). The PK-PD model that includes a large effect compartment requires intercompartmental transport rate constants different from those for the small volume of the effect compartment (Table 1 ). The peak receptor occupancy, Occ $=\mathrm{Occ}_{\mathrm{NMB} 50}=0.875$, and the peak $[\mathrm{D}]_{\mathrm{e}}=\mathrm{IC50}$ $=7 \cdot \mathrm{K}_{\mathrm{D}^{\prime}}$, were attained at $4.50 \mathrm{~min}$ for either volume of the effect compartment. Hence, for both volumes the simulated peak $\mathrm{NMB}=\mathrm{NMB} 50$ and occurs at $4.5 \mathrm{~min}$ after injection, but the time course of $\mathrm{NMB}$ is different between the small and large volumes of the effect compartment (lower panel in Figure 1). To reach the respective peaks at 4.50 min after the injection required $k_{e 1}$ that was approximately four times higher for the small than for the large effect compartment (Table 1).

The calculations were verified by calculating the sum of the amounts in the four compartments plus the amount eliminated from the body. For all times between 0 and 50 min after injection, the sum was equal to ED50. Expressed as fractions of the administered dose (= ED50), the peak amounts in compartment ${ }_{2}$ and compartment cond $_{3}$ and the times after injection when the peaks were attained are for the small volume of the effect compartment 0.199 at 1.6 min and 0.483 at $7.3 \mathrm{~min}$, respectively. For the large volume, the corresponding values are 0.158 at $1.3 \mathrm{~min}$ and 0.268 at $11.9 \mathrm{~min}$.

Two additional observations were made during these simulations. First, exclusion of the small effect compartment from the PK-PD model only minimally influences the fit of the amounts in compartment ${ }_{1}$ to the target plasma amounts. The result is not unexpected, because the intercompartmental transport rate constants (microconstants) in the model with a small volume of the effect compartment (Table 1) are close to those in the standard 3-compartment model. Second, when the effect compartment in the PK-PD model was postulated not to contain the receptors, i.e., $\{\mathrm{R}\}_{\text {total }}=0$, identical values of $\mathrm{k}_{\mathrm{e} 1}$ establish the peak free amount of $\mathrm{D}$ in the respective effect compartment at identical times (data not presented).

Based on the derived pharmacokinetic rate constants, $\mathrm{NMB}$ was simulated with different doses of $\mathrm{D}$. One thousand points were selected for a 10-fold increase in doses. 


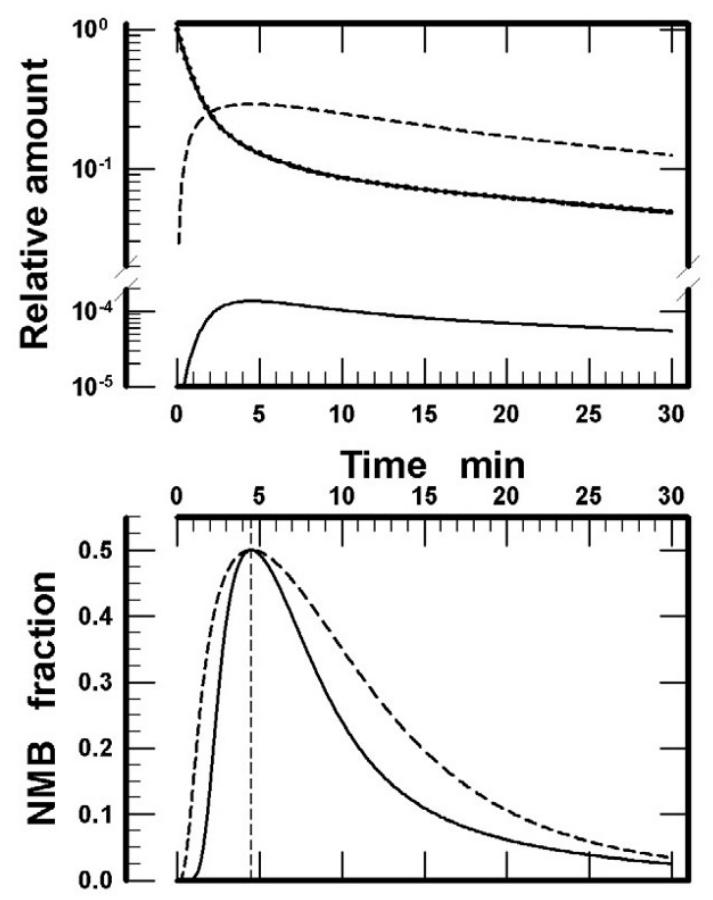

\section{Figure I}

Upper panel: The amounts of muscle relaxant $D\left(K_{D}=1 \cdot 10^{-7}\right.$ $\mathrm{M})$ in plasma and compartment ${ }_{1}$ and the free amounts in the effect compartment assigned a small $\left(\mathrm{V}_{\mathrm{e}}=4.4 \cdot 10^{-5} \mathrm{~L} \cdot \mathrm{kg}^{-1}\right)$ or a large $\left(\mathrm{V}_{\mathrm{e}}=9.23 \cdot 10^{-2}{\left.\mathrm{~L} \cdot \mathrm{kg}^{-1}\right)}\right)$ volume. All the amounts are normalized to the injected dose $\left(=\operatorname{ED} 50=2.23 \cdot 10^{-7} \mathrm{~mol} \cdot \mathrm{kg}^{-1}\right)$.

Solid and dashed lines indicate the amounts contained in the small and the large effect compartment, respectively. Filled circles denote the target amounts in plasma defined by the triexponential function. The three curves for the amounts in plasma overlap. The estimates were obtained at $0.1 \mathrm{~min}$ intervals. Lower panel: Time course of the neuromuscular block (NMB) by ED50 of the muscle relaxant D. NMB was calculated using Eq I (Appendix), [D] $]_{e}$ for the small and large volume of the effect compartment presented in the upper panel, and by setting $\gamma=4.0$ and IC50 $=7 \cdot 10^{-7} \mathrm{M}$. The lines are identical to those in the upper panel for the small and large volume of the effect compartment.

$\mathrm{NMB}$ was calculated with the peak free concentrations of $\mathrm{D}$ in the effect compartment using Eq 1 in the Appendix $\left(\gamma=4\right.$ and IC50 $\left.=7 \cdot 10^{-7} \mathrm{M}\right)$. The relationship between $\mathrm{NMB}$ and the doses that produced the peak concentrations differed between the models (upper panel in Figure 2 for $\mathrm{NMB}=0.05$ to $\mathrm{NMB}=0.95$, i.e., $\mathrm{NMB} 05$ to $\mathrm{NMB} 95$ ). To obtain a quantitative estimate for the difference, equation of Hill (Eq 2) was fitted to both sets of points to describe the relationship between NMB and the injected
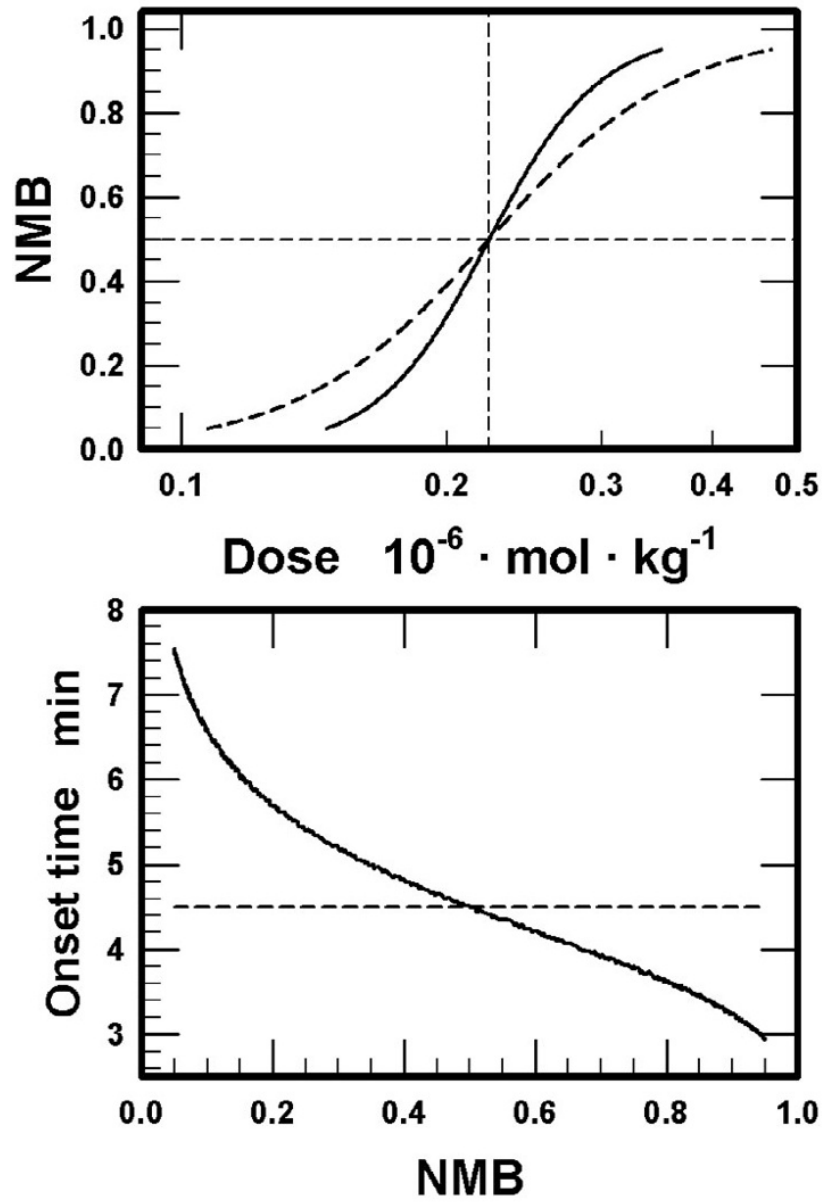

Figure 2

Upper panel: Neuromuscular block (NMB) calculated as a function of the peak concentrations of muscle relaxant $D$ in the effect compartment using Eq I presented in the Appen$\operatorname{dix}\left(\gamma=4.0\right.$ and IC50 $\left.=7 \cdot 10^{-7} \mathrm{M}\right)$. The doses presented along the abscissa refer to the doses that established the peak concentrations. The range of NMB is from NMB05 to NMB95. One thousand logarithmically equidistant values were used for a 10 -fold increase in doses. The volumes of the effect compartment and the lines are identical to those presented in Figure I. Lower panel: Onset times as a function of the magnitude of NMB. Onset times are defined as the times after the bolus intravenous injection of muscle relaxant $D$ needed to establish peak NMB, from NMB05 to NMB95. Other details are identical to those in the upper panel.

doses. The fit was excellent for both sets $\left(\mathrm{r}^{2}>0.9999\right.$, the number of points, $n,=381$ for the small and $n=641$ for the large volume of the effect compartment). The $95 \%$ confidence interval $(95 \% \mathrm{CI})$ for the fitted $\gamma_{\mathrm{f}}$ was 6.819 to 


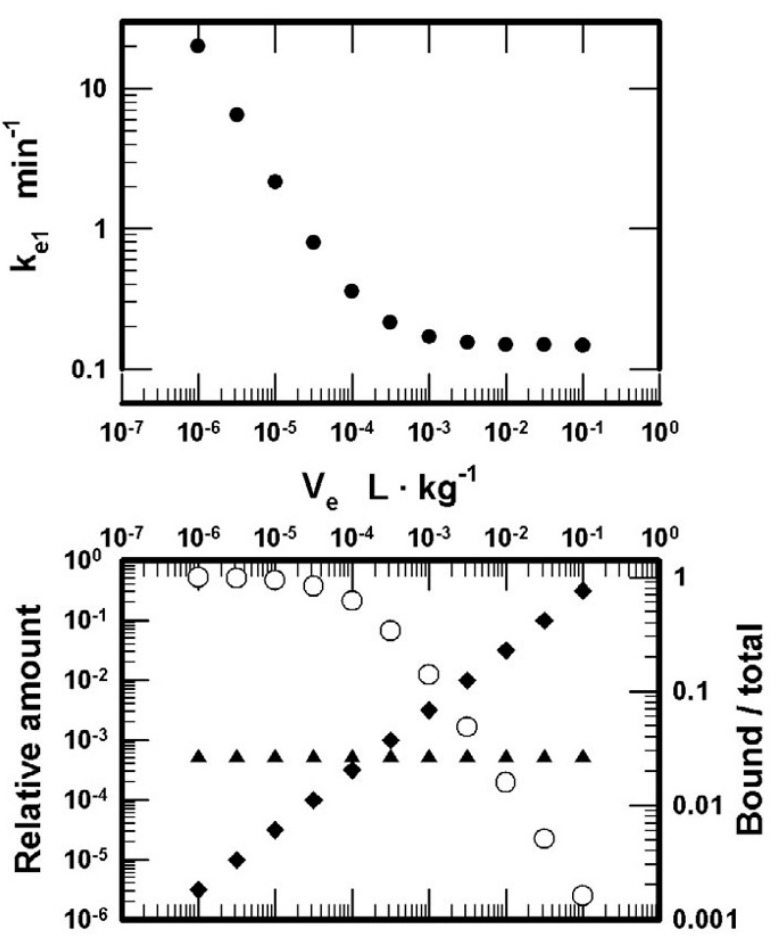

\section{Figure 3}

Upper panel: Values estimated for the transport rate constant between the effect and the central compartment, $k_{e l}$, as a function of the volume assigned to the effect compartment, $\mathrm{V}_{\mathrm{e}}$. The dose of the muscle relaxant $\mathrm{D}=\mathrm{ED} 50=2.23 \cdot 10^{-7}$ $\mathrm{mol} \cdot \mathrm{kg}^{-1}$ and $K_{D}=1 \cdot 10^{-7} \mathrm{M}$. Lower panel: Amounts of the muscle relaxant $D$ (left $Y$-axis) bound to the receptors (filled upright triangles) and the amounts free (filled diamonds). The amounts are normalized to the injected dose presented in the upper panel. The ratio of the bound to the total amounts (empty circles; total = bound + free; right $Y$-axis) in the effect compartment is presented as a function of the volume assigned to the effect compartment.

6.838 for the small and 4.0040 to 4.0041 for the large effect compartment. The $95 \% \mathrm{CI}$ for the fitted $\mathrm{ED} 50_{\mathrm{f}}$ were $(2.235$ to 2.236$) \cdot 10^{-7} \mathrm{~mol} \cdot \mathrm{kg}^{-1}$ and $(2.23256$ to $2.23258) \cdot 10^{-7} \mathrm{~mol} \cdot \mathrm{kg}^{-1}$, respectively.

The onset times for NMB05 to NMB95 differed between the models assigned different volumes of the effect compartment (lower panel in Figure 2). The model with the large effect compartment projected that the onset times were nearly independent of the magnitude of NMB. The model incorporating a small volume of the effect compartment projected an inverse relationship between the onset times and the magnitudes of NMB. For $\mathrm{NMB}<$ NMB50, the onset times were longer, and for $\mathrm{NMB}>$ NMB50 the onset times were shorter than those projected by the model with a large effect compartment.

\section{Simulations with different volumes assigned to the effect compartment using ED50}

Next, the influence of the volume assigned to the effect compartment was examined systematically. The volumes varied from $1 \cdot 10^{-6}$ to $1 \cdot 10^{-1} \mathrm{~L} \mathrm{~kg}^{-1}(11$ logarithmically equidistant values). The pharmacokinetic parameters, including $\mathrm{k}_{\mathrm{e} 1}$, were estimated as previously stipulated, i.e., ED50 dose establishes peak receptor occupancy = Occ875 and peak $[\mathrm{D}]_{\mathrm{e}}=\mathrm{IC} 50$ at $4.5 \mathrm{~min}$ after injection. The coefficient of variation for the fit of the concentrations of $D$ in compartment $_{1}$ to the target plasma concentrations was $\mathrm{CV}$ $=0.84 \%$ for the largest and $=0.0006 \%$ for the smallest volume. The values for $\mathrm{k}_{\mathrm{e} 1}$ as a function of the assigned volumes, estimated with ED50 and using the same muscle relaxant $\left(K_{D}=1 \cdot 10^{-7} \mathrm{M}\right)$, are presented in the upper panel of Figure 3. The results demonstrate that $k_{e 1}$ increases markedly for the smaller values of $V_{e}$. The relative amounts of D bound to the receptors, the amounts free in the effect compartment, and the ratio of the bound to the total amount in the effect compartment with ED50 show (lower panel in Figure 3) that for all volumes the amounts of $\mathrm{D}$ bound to the receptors are constant. For smaller volumes the bound amounts make up nearly all of D present in the effect compartment, while for larger volumes the total amount of D is nearly completely accounted for by the free amount.

\section{Simulations using different volumes and different doses}

We used the set of pharmacokinetic parameters obtained for each assigned volume, but now varied the dose using 1000 values for a ten-fold increase. The results are presented in Figure 4. Increasing doses increase the peak free concentrations of D for each volume of the effect compartment (upper panel in Figure 4). The increase is steepest for the smallest volume and the slopes decrease for the larger assigned volumes. The estimated peak free concentrations of D in the effect compartment were used to calculate NMB (IC50 $=7 \cdot 10^{-7} \mathrm{M}$ and $\gamma=4$, Eq 1 in Appendix). The values of NMB from NMB05 to NMB95 as calculated using $[\mathrm{D}]_{e}$ were plotted against the injected doses separately for each assigned volume, similarly to the results presented in the upper panel of Figure 2 . The modified equation of Hill (Eq 2, Appendix) was fitted to each of these 11 sets of points to define $\mathrm{NMB}$ as a function of the injected doses. The fit was excellent $\left(r^{2}>0.9996\right.$ for $n$ between 314 to 641 points). The fitted values of $\gamma_{\mathrm{f}}$ are presented in the lower panel in Figure 4. The values increase markedly for smaller volumes. The $95 \% \mathrm{CI}$ for the eleven fitted estimates of $\mathrm{ED} 0_{\mathrm{f}}$ varied between (2.225 to $2.226) \cdot 10^{-7} \mathrm{~mol} \cdot \mathrm{kg}^{-1}$ for the smallest and $(2.25720$ to 

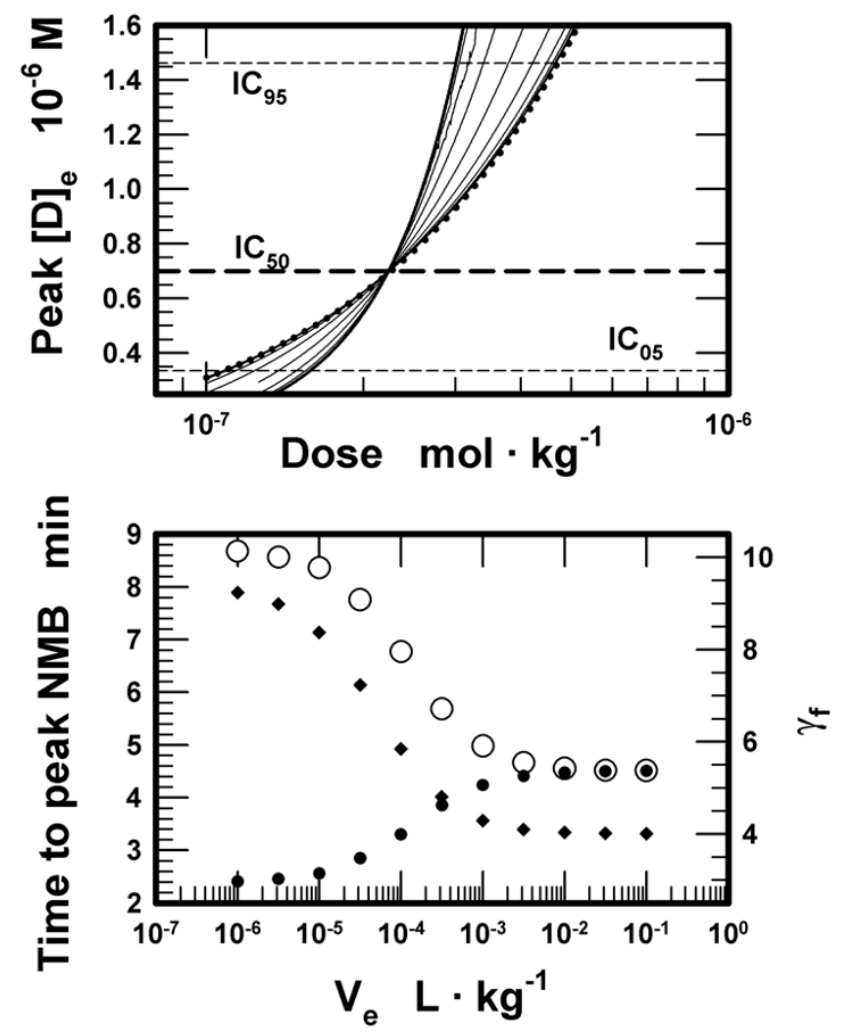

\section{Figure 4}

Upper panel: The peak free concentrations of muscle relaxant $D$ in the effect compartment calculated with variable doses of $D$ as a function of the volumes assigned to the effect compartment. The assigned volumes were: $1 \cdot 10^{-6}, 3.16 \cdot 10^{-6}, 1 \cdot 10^{-}$ 5, 3.16 $10^{-5}, 1 \cdot 10^{-4}, 3.16 \cdot 10^{-4}, 1 \cdot 10^{-3}, 3.16 \cdot 10^{-3}, 1 \cdot 10^{-2}, 3.16 \cdot 10^{-}$ 2 , and $1 \cdot 10^{-1} \mathrm{~L}_{\mathrm{kg}^{-1}}$. The bold solid and the dotted lines indicate the lowest and the highest assigned volumes, respectively. Concentrations for the intermediate volumes are indicated in sequence by thin solid lines. The three dashed lines parallel with the $\mathrm{X}$-axis represent the free concentration of D for NMB95 (IC95, upper line), for NMB50 (IC50, middle line) and for NMB05 (IC05, lower line). The concentrations for IC05 and IC95 were calculated based on $\gamma=4.0$ (Eq I, Appendix). Lower panel: Times to NMB05 (open circles) and NMB95 (filled circles, left $Y$-axis) as a function of the volumes assigned to the effect compartment, $V_{e}$. Neuromuscular block was calculated using Eq I (IC50 $=7 \cdot 10^{-7} \mathrm{M}$, $\gamma=4)$ and the peak free concentrations of $D$ presented in the upper panel. The values of the exponent $\gamma_{f}$ (filled diamonds, right $Y$-axis) were obtained by fitting Eq 2 to the calculated NMB.

$2.25722) \cdot 10^{-7} \mathrm{~mol} \cdot \mathrm{kg}^{-1}$ for the largest volume. These simulations permitted us to estimate the times to NMB05 and
NMB95 (onset times). The results are presented in the lower panel of Figure 4. Onset times for NMB05 and NMB95 differ widely for the small volumes, but the differences progressively decrease for larger volumes of the effect compartment. The onset times for NMB05 and NMB95 are nearly identical for the largest assigned volume.

\section{Simulations with different binding affinities assigned to muscle relaxants}

The PK-PD model was also tested with two additional muscle relaxants using the previously defined small and large volumes of the effect compartment. One muscle relaxant, $\mathrm{D}_{2}$, was assigned a 10 times lower affinity for the binding sites at the receptors, $\mathrm{K}_{\mathrm{D} 2}=1 \cdot 10^{-6} \mathrm{M}$. The other, $\mathrm{D}_{3}$, was assigned a 10 times higher affinity, $\mathrm{K}_{\mathrm{D} 3}=1 \cdot 10^{-8}$ $\mathrm{M}$. The assignments changed the respective $\mathrm{k}_{\mathrm{diss}}$, but not $\mathrm{k}_{\mathrm{assoc}}$. Two series of simulations were performed. In the first series, all the pharmacokinetic constants, including $\mathrm{k}_{\mathrm{e} 1}$, were those defined previously for either the small or the large volume of the effect compartment and for the muscle relaxant with $\mathrm{K}_{\mathrm{D}}=1 \cdot 10^{-7} \mathrm{M}$ (Table 1 ). A 100-fold increase in affinities was projected to require 16.3 times lower ED50 for the small volume $\left(\mathrm{ED} 50=1.144 \cdot 10^{-6}\right.$ $\mathrm{mol} \cdot \mathrm{kg}^{-1}$ for $\mathrm{D}_{2}$ and ED50 $=7.018 \cdot 10^{-8} \mathrm{~mol} \cdot \mathrm{kg}^{-1}$ for $\left.\mathrm{D}_{3}\right)$, but a 98.9 times lower ED50 $\left(\operatorname{ED} 50=2.230 \cdot 10^{-6} \mathrm{~mol} \cdot \mathrm{kg}^{-}\right.$ ${ }^{1}$ for $\mathrm{D}_{2}$ and $\mathrm{ED} 50=2.255 \cdot 10^{-8} \mathrm{~mol} \cdot \mathrm{kg}^{-1}$ for $\mathrm{D}_{3}$ ) for the model with a large volume of the effect compartment. For the small effect compartment, the times to reach NMB50 were $1.82 \mathrm{~min}$ for $\mathrm{D}_{2}$ and $34.74 \mathrm{~min}$ for $\mathrm{D}_{3}$. In the model with a large effect compartment, the times to NMB50 differed only minimally, from $4.50 \mathrm{~min}$ for $\mathrm{D}_{2}$ to $4.54 \mathrm{~min}$ for $\mathrm{D}_{3}$.

In the second series of simulations, we postulated that ED50 of either $\mathrm{D}_{2}$ or $\mathrm{D}_{3}$ produces $\mathrm{NMB} 50$ at $4.5 \mathrm{~min}$ after injection using either the small or the large volume of the effect compartment. The doses producing NMB50 were related to the assigned $\mathrm{K}_{\mathrm{D}}$ values, i.e., $\mathrm{ED} 50=2.2325 \cdot 10^{-}$ ${ }^{6} \mathrm{~mol} \cdot \mathrm{kg}^{-1}$ for $\mathrm{D}_{2}$ and $\mathrm{ED} 50=2.2325 \cdot 10^{-8} \mathrm{~mol} \cdot \mathrm{kg}^{-1}$ for $\mathrm{D}_{3}$. These doses establish plasma concentrations at 4.5 $\min [\mathrm{D}]_{\text {plasma }}=\mathrm{IC50}=7 \cdot \mathrm{K}_{\mathrm{D}}=$ peak $[\mathrm{D}]_{\mathrm{e}}$. In the model containing a small volume of the effect compartment, the postulate was satisfied by $\mathrm{k}_{\mathrm{e} 1}=0.196 \mathrm{~min}^{-1}$ for $\mathrm{D}_{2}$ and $\mathrm{k}_{\mathrm{e} 1}$ $=4.710 \mathrm{~min}^{-1}$ for $\mathrm{D}_{3}$. For the large volume, the estimates of $\mathrm{k}_{\mathrm{e} 1}$ were $0.1475 \mathrm{~min}^{-1}$ for $\mathrm{D}_{2}$ and $0.1498 \mathrm{~min}^{-1}$ for $\mathrm{D}_{3}$.

\section{Discussion}

The simulations suggest that the volume of the effect compartment per se is not the critical parameter in a PK-PD model for nondepolarizing muscle relaxants. If the effect compartment is postulated to be void of the postsynaptic receptors, then the peak concentration of free muscle relaxant in this compartment is attained at identical times using identical transport rate constant $\mathrm{k}_{\mathrm{e} 1}$ for any volume 
of the effect compartment. These conclusions agree with those obtained from PK-PD models assuming a negligibly small volume of the effect compartment and not taking into account binding of a muscle relaxant to the postsynaptic receptors [1-3]. However, NMB is produced not by the free molecules of muscle relaxants in the effect compartment, but by the molecules bound to the postsynaptic receptors at the motor end plates. Therefore, consideration of binding of muscle relaxants to the postsynaptic receptors in the effect compartment is advantageous in PK-PD modeling. The present simulations confirm the conclusion from the reports [4-6] that the receptor concentration in the effect compartment is a critical parameter in PK-PD modeling. The predictions from our simulations assuming a low or a high receptor concentration differ with respect to (1) the onset times to the peak but submaximal neuromuscular block for a single muscle relaxant (lower panel in Figure 2), (2) the time course of NMB using ED50 (lower panel in Figure 1), (3) the shape of the NMB-versus-dose curves (upper panel in Figure 2), (4) the estimates of $k_{e 1}$ (Table 1 and upper panel in Figure 3 ), and (5) the estimates of ED50 and the onset times as a function of affinities assigned to the muscle relaxants for binding to the receptors $\left(\mathrm{D}_{2}\right.$ and $\mathrm{D}_{3}$, Results).

In the aforementioned models [4-6], receptor concentration was an explicit model parameter. In contrast, the present model defines the receptor concentration as the ratio between the constant amount of postsynaptic receptors and the variable volume assigned to the effect compartment. This approach allows PK-PD modeling without the constraint of a negligibly small effect compartment. The earlier models taking into account receptor concentration [4-6] assumed a negligibly small volume of the effect compartment. Given a fixed amount of postsynaptic receptors, a finite receptor concentration is not compatible with the negligibly small volume of the effect compartment. This is an inherent weakness of such models.

A compartment is defined by Jacquez [12] as "an amount of a material that acts kinetically like a distinct, homogenous amount". This is the reason that the five equations defining the transport and the distribution of a muscle relaxant in the body (Appendix) were formulated in terms of amounts rather than concentrations. The total amount of a drug in the body is represented by two, three, or more such compartments. The necessity to invoke more than a single compartment arises from the physicochemical properties of the drug in relation to those structures in the body that prevent drug's uniform dilution. Anatomical structures and/or physiologic processes represent these barriers. For muscle relaxants, small hydrophilic cations with $\mathrm{MW}<1000 \mathrm{da}$, the principal barriers are the capillary wall and the cellular membranes. It seems plausible to postulate that muscle relaxants dif- fuse through the pores in the capillary wall into the surrounding interstitial spaces. Diffusion across the cellular membranes is very unlikely due to the high hydrophilicity of the molecules. Therefore and as a first approximation, muscle relaxants remain diluted in a space limited to plasma and the interstitial space. The pharmacokinetic compartments for muscle relaxants likely represent the amounts of muscle relaxants in plasma and the interstitial spaces of different tissues.

In the muscle, muscle relaxants diffuse throughout the interstitial space, including the synaptic clefts at the motor end plates. There are no anatomical barriers between the interstitial space in muscle and the synaptic clefts to prevent diffusion of muscle relaxants into the synaptic clefts [13]. These considerations qualify the interstitial space in muscle, including the synaptic spaces, as a single pharmacokinetic compartment. The volume of the interstitial space in muscle defines the volume of this compartment.

Due to the presence of the postsynaptic receptors in the synaptic clefts, the

compartment represents the effect compartment for muscle relaxants. The functional receptors are immobile and are located exclusively within the synaptic clefts. Hence, interaction between the receptors and the free molecules of a muscle relaxant occurs due to diffusion of the free molecules of the muscle relaxant to the receptors. In effect, interaction between the two partners may be represented as proceeding in a space common to both, i.e., the interstitial space in muscle. Volume of this space defines the volume of the effect compartment, $\mathrm{V}_{\mathrm{e}}$. We suggest that the apparent or the effective concentration of the postsynaptic receptors for the interaction with muscle relaxants is the ratio of the amount of receptors and the volume of interaction, $[\mathrm{R}]_{\text {total }}=\{\mathrm{R}\}_{\text {total }} / \mathrm{V}_{\mathrm{e}}$.

Transport of a drug between two compartments is represented in a standard pharmacokinetic model by two firstorder rate constants. A modification of this approach is needed, if the transport is assumed to proceed via diffusion. Occurrence of a peak amount in a non-central compartment suggests that at that moment there is no net transport. The postulate of transport via diffusion implies that the concentration of a muscle relaxant in the central and the peak concentration in the effect compartment are identical at that moment. In the simulations, the transport rate constant out of the effect compartment into compartment $_{1}$ is represented by the symbol $\mathrm{k}_{\mathrm{e} 1}$. The rate constant in the opposite direction, $\mathrm{k}_{1 e}$ is expressed as a function of $\mathrm{k}_{\mathrm{e} 1}$, viz., $\mathrm{k}_{1 e}=\mathrm{k}_{\mathrm{e} 1} \cdot\left(\mathrm{V}_{\mathrm{e}} / \mathrm{V}_{1}\right)$. The expression results from the postulate that the transport occurs via diffusion. We suggest that $\mathrm{k}_{\mathrm{e} 1}$ may be interpreted as the ratio 
of the plasma flow to the muscle and the volume of the interstitial space in muscle. For the adductor pollicis muscle and assuming plasma flow to the forearm or the hand of 0.9 to $4.7 \mathrm{~mL} \cdot \mathrm{min}^{-1} \cdot(100 \mathrm{~g} \text { muscle })^{-1}[14]$ and the volume of the interstitial space in muscle of 15 to 22 $\mathrm{mL} \cdot(100 \mathrm{~g} \text { muscle })^{-1}$, the value of the transport rate constant $\mathrm{k}_{\mathrm{e} 1}$ may be estimated to between 0.041 and $0.313 \mathrm{~min}^{-1}$.

The postulate seems plausible that the molar amount of the postsynaptic receptors is a physiologic constant. The value of the constant may be 10 times lower or 10 times higher than the assigned value (Table 1) without markedly altering the results of the simulations. The postulate of a constant amount of postsynaptic receptors permits the definition of the apparent receptor concentration in the effect compartment via the relationship $[\mathrm{R}]_{\text {total }}=$ $\{\mathrm{R}\}_{\text {total }} / \mathrm{V}_{\mathrm{e}}$.

The results of the simulations demonstrate that a PK-PD model may be constructed for a wide range of volumes assigned to the effect compartment. We examined $V_{e}$ from $1 \cdot 10^{-6}$ to $1 \cdot 10^{-1} \mathrm{~L}^{\mathrm{kg}} \mathrm{kg}^{-1}$ and the corresponding apparent concentrations of the receptors. In general, smaller volumes require higher values of $k_{e 1}$ (upper panel in Figure 3), are associated with smaller total amounts of the muscle relaxant in the effect compartment and larger fractions of the muscle relaxant in the bound form (lower panel in Figure 3). The smaller volumes are compatible with the intercompartmental transport rate constants close to those in the standard 3-compartment pharmacokinetic model. For volumes $<1 \cdot 10^{-3} \mathrm{~L} \cdot \mathrm{kg}^{-1}$, the onset times of submaximal $\mathrm{NMB}$ are negatively correlated with the magnitude of NMB (lower panels in Figures 2 and 4). The onset times are also markedly dependent on the values assigned to the equilibrium dissociation constants for binding of the muscle relaxants to the receptors (muscle relaxants $\mathrm{D}_{2}$ and $\mathrm{D}_{3}$ ), higher affinities associated with prolonged onset times. All these findings change for $\mathrm{V}_{e}>$ $1 \cdot 10^{-3} \mathrm{~L} \cdot \mathrm{kg}^{-1}$ and the receptors concentrations $<1 \cdot 10^{-7} \mathrm{M}$ (Figures 3 and 4). Specifically, the values of the rate constant $k_{\mathrm{e} 1}$ become smaller and relatively independent of the assigned volumes (upper panel in Figure 3), the differences between onset times for NMB05 and NMB95 progressively disappear (lower panel in Figure 4), the affinities do not influence the onset to NMB50, and ED50 doses are proportional to $K_{D}$ (muscle relaxants $D_{2}$ and $D_{3^{\prime}}$ Results). It appears as if the value of $\mathrm{V}_{e}$ of about $1 \cdot 10^{-3}$ $\mathrm{L} \cdot \mathrm{kg}^{-1}$ and the receptor concentration $\sim 1 \cdot 10^{-7} \mathrm{M}$ represent the critical threshold for the difference between a "small" and a "large" volume of the effect compartment.

The results of the simulations reveal a difference in the slopes of the NMB curves when evaluated as a function of the injected doses of a muscle relaxant. As in the available pharmacodynamic models, NMB in the proposed model was calculated using the peak free concentration of a muscle relaxant in the effect compartment and two constants: $\gamma$ and IC50 (Eq 1, Appendix). When the NMB, calculated using peak $[D]_{e^{\prime}}$, was plotted as a function of the doses that produced these peak concentrations in the effect compartment, the fitted value of $\gamma_{\mathrm{f}}$ (Eq 2, Appendix) was larger than $\gamma$ used in the calculation of $\mathrm{NMB}$ from $[\mathrm{D}]_{e}$ (upper panel in Figure 2 and lower panel in Figure 4) and the fitted values of $\gamma_{\mathrm{f}}$ increase progressively for smaller volumes assigned to the effect compartment (lower panel in Figure 4). For volumes $>10^{-3} \mathrm{~L} \cdot \mathrm{kg}^{-1}$, the fitted values of $\gamma_{\mathrm{f}}$ approach the value of $\gamma$ used in the calculations of $\mathrm{NMB}$ from $[D]_{e}$ (lower panel, Figure 4). The difference is due to the relationship between the peak concentrations of the free muscle relaxant in the effect compartment and the injected doses (upper panel in Figure 4). For volumes < $10^{-3} \mathrm{~L} \cdot \mathrm{kg}^{-1}$, the peak concentrations increase rapidly with increasing doses. The steeper slope implies that the difference in doses producing IC05 and IC95, corresponding to NMB05 and NMB95, respectively, is smaller the smaller the volume assigned to the effect compartment. The narrower spread of these doses leads, in turn, to higher fitted values of $\gamma_{\mathrm{f}}$ when NMB is represented as a function of the injected dose. To summarize, if $\mathrm{V}_{e}<10^{-3} \mathrm{~L} \cdot \mathrm{kg}^{-1}$, then a correlation of $\mathrm{NMB}$ to the doses needed to establish the peak concentrations requires fitted values for $\gamma_{\mathrm{f}}$ higher than the value of $\gamma$ used in calculating NMB from $[D]_{e}$. For $\mathrm{V}_{\mathrm{e}}>10^{-}$ ${ }^{3} \mathrm{~L} \cdot \mathrm{kg}^{-1}$, the estimates of the fitted $\gamma_{\mathrm{f}}$ approach the value of $\gamma$ used in calculating NMB as a function of $[D]_{e}$. Therefore, a comparison of $\gamma$, estimated in a PK-PD model and based on $[D]_{e^{\prime}}$ with $\gamma_{f^{\prime}}$ obtained experimentally in a NMB-versusdose study, provides information about the volume of the effect compartment and the receptor concentration in it. The fitted values of $\mathrm{ED} 5 \mathrm{f}_{\mathrm{f}}$ are rather independent of the volumes assigned to the effect compartment and the estimates are close to the a priori defined ED50 used in calculating the target plasma concentrations.

The PK-PD models are based on two sets of experimental data: the time course of the plasma concentration of a muscle relaxant and the time course of NMB. The models simulate, and are applicable only to, the concentrations in plasma and in the postulated effect compartment. The amounts or concentrations in the other compartments and the amount eliminated from the body are not verifiable from the available experimental data. These compartments are included in the current PK-PD model solely to preserve mass balance and to fit the amounts or concentrations of $\mathrm{D}$ in compartment ${ }_{1}$ to the target plasma amounts or concentrations. A posteriori addition of a large effect compartment to the standard 3-compartment PK model alters the simulated amounts or concentrations in compartment $_{1}$ and the fit of the standard 3-compartment model to the target plasma concentrations is lost. 
Realization of this fact was the primary reason for the postulate of a negligibly small effect compartment in the previously introduced PK-PD model [2]. However, as demonstrated in the current simulations, a PK-PD model may include an effect compartment of any volume and contain a sizable fraction of the injected dose, if the model is designed $a$ priori and the pharmacokinetic rate constants, including $\mathrm{k}_{\mathrm{e} 1}$, adjusted so that the amounts in

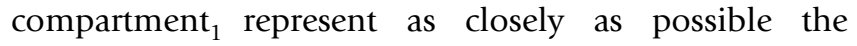
observed amounts in plasma. The fitting process is similar to that for fitting a standard pharmacokinetic model to the observed plasma concentrations. Alternatively and without prejudging mass transport from plasma to any compartment, the amounts in plasma may be described using a multiexponential equation without detriment to the pharmacodynamic part of the model.

\section{Conclusion}

The simulations do not indicate whether a PK-PD model containing a small or a large effect compartment is more appropriate. The selection should be based on the results of prospective clinical experiments. The simulations suggest an optimal experimental design. The study needs to be conducted with several muscle relaxants. Several doses of each are selected to produce less than complete NMB, e.g., NMB10 to NMB90. The experiment needs to answer the following question: Is the onset time of submaximal NMB produced by a single muscle relaxant a function of the level of NMB? If the results with a single muscle relaxant show an inverse relationship between the level of $\mathrm{NMB}$ and the onset times, then the model containing a small volume of the effect compartment and a high receptor concentration is more appropriate. If the onset times are independent of the magnitude of the submaximal $\mathrm{NMB}$, then the PK-PD model containing a large volume of the effect compartment and a low receptor concentration is more appropriate.

\section{Appendix}

The pharmacokinetic part of the model was formulated with the volume of the effect compartment, $\mathrm{V}_{\mathrm{e}^{\prime}}$ explicitly incorporated in the model. The following symbols are used: $\mathrm{D}$ for the muscle relaxant and $\mathrm{R}$ for the receptors. The braces denote molar amounts per kg body weight. The first and second subscript appended to the rate constants denote the number of the source and the target compartments, respectively. Subscript $e$ denotes the effect compartment, e.g., $\mathrm{k}_{1 e}$ denotes the rate constant for the transport from compartment ${ }_{1}$ to the effect compartment and $\mathrm{k}_{\mathrm{e} 1}$ the transport in the reverse direction. The symbol $\{D\}_{e}$ denotes the free amount of $D$ in the effect compartment.

$$
\begin{aligned}
& \frac{d}{d t}\{D\}_{1}=-\left(k_{10}+k_{12}+k_{13}+k_{1 e}\right) \cdot\{D\}_{1} \\
& +k_{21} \cdot\{D\}_{2}+k_{31} \cdot\{D\}_{3}+k_{e 1} \cdot\{D\}_{e} \\
& \frac{d}{d t}\{D\}_{2}=k_{12} \cdot\{D\}_{1}-k_{21} \cdot\{D\}_{2} \\
& \frac{d}{d t}\{D\}_{3}=k_{13} \cdot\{D\}_{1}-k_{31} \cdot\{D\}_{3} \\
& \frac{d}{d t}\{D\}_{e}=k_{1 e} \cdot\{D\}_{1}-k_{e 1} \cdot\{D\}_{e}-\frac{d}{d t}\{D R\} \\
& \frac{d}{d t}\{D R\}=\frac{k_{\text {assoc }}}{V_{e}} \cdot\{D\}_{e} \cdot\left(\{R\}_{\text {total }}-\{D R\}\right)-k_{\text {diss }} \cdot\{D R\}
\end{aligned}
$$

DR represents the $1: 1$ complex of $\mathrm{D}$ with the receptors within the effect compartment. The differential equation for $\{D R\}$ was derived from the differential equation for [DR] written in terms of the molar concentrations $[D]_{\mathrm{e}^{\prime}}$ $[R]_{\text {total }^{\prime}}$ and [DR]. Multiplication of this equation by $\mathrm{V}_{e}$ converts the concentrations into amounts. The definition of $\mathrm{k}_{1 e}$ in terms of $\mathrm{ke1}_{1}, v i z ., \mathrm{k}_{1 e}=\mathrm{k}_{\mathrm{e} 1} \cdot\left(\mathrm{V}_{\mathrm{e}} / \mathrm{V}_{1}\right)$, results from the postulate of diffusion as the transport mechanism and implies that the peak concentration of $\mathrm{D}$ in the effect compartment equals the concentration in compartment ${ }_{1}$ at the same moment. The initial conditions at $t=0$ are: $\{D\}_{1}=$ dose, and $\{D\}_{2}=\{D\}_{3}=\{D\}_{e}=\{D R\}=0$.

The Hill equation for the calculation of NMB from the free molar concentrations of the muscle relaxant $\mathrm{D}$ in the effect compartment, $[D]_{e^{\prime}}$ is given by:

$N M B=\frac{[D]_{e}^{\gamma}}{[D]_{e}^{\gamma}+I C 50^{\gamma}} \quad \mathbf{E q 1}$

where $[D]_{e}=$ peak $\{D\}_{e} / V_{e}$ and IC50 $=7 \cdot K_{D}=$ peak $[D]_{e}$ when Occ $=0.875$. The exponent $\gamma$ was arbitrarily assigned a value of 4.0 .

A different form of the Hill equation was used to fit the calculated NMB (Eq.1) as a function of the doses producing the peak $[D]_{\mathrm{e}}$. The modified equation relates $\mathrm{NMB}$ to the injected doses:

$N M B=\frac{d o s e^{\gamma_{f}}}{d o s e^{\gamma_{f}}+E D 50_{f}^{\gamma_{f}}}$

Eq2

The values for the exponent $\gamma_{\mathrm{f}}$ and $\mathrm{ED} 50_{\mathrm{f}}$ were derived in the fitting process.

\section{Competing interests}

The author(s) declare that they have no competing interests. 


\section{Authors' contributions}

All authors have contributed equally to the conception and the design of simulations, to acquisition of data and data analysis, and to the preparation of the manuscript. All authors read and approved the final manuscript.

\section{Note}

${ }^{1} \mathrm{CV} \mathrm{in} \%=100 \cdot \sqrt{\frac{\sum_{i=1}^{n}\left(\ln \left[D_{1}\right]_{i}-\ln \left[D_{\text {plasma }}\right]_{i}\right)^{2}}{n-1}}$, where

$\left[\mathrm{D}_{\text {plasma }}\right]_{\mathrm{i}}$ is the molar concentration of $\mathrm{D}$ in plasma calculated from the triexponential equation at time $t_{i},\left[D_{1}\right]_{i}$ is the molar concentration in compartment cot $_{1}$ at the same time, and $\mathrm{n}$ is the number of time points $(=300)$.

\section{Acknowledgements}

A.A. is grateful to the Bernhard-Lang Research Association for continuous support.

\section{References}

I. Hull CJ, Van Beem HB, McLeod K, Sibbald A, Watson MJ: A pharmacodynamic model for pancuronium. $\mathrm{Br} J$ Anaesth 1978, 50: III3-II23.

2. Sheiner LB, Stanski DR, Vozeh S, Miller RD, Ham J: Simultaneous modeling of pharmacokinetics and pharmacodynamics: application to d-tubocurarine. Clin Pharmacol Ther 1979, 25:358-37I.

3. Laurin J, Donati F, Nekka F, Varin F: Peripheral link model as an alternative for pharmacokinetic-pharmacodynamic modeling of drugs having a very short elimination half-life. J Pharmacokinet Biopharm 200I, 28:7-25.

4. De Haes A, Proost JH, Kuks JB, van den Tol DC, Wierda JM: Pharmacokinetic/pharmacodynamic modeling of rocuronium in myasthenic patients is improved by taking into account the number of unbound acetylcholine receptors. Anesth Analg 2002, 95:588-596.

5. Donati F, Meistelman C: A kinetic-dynamic model to explain the relationship between high potency and slow onset time for neuromuscular blocking drugs. J Pharmacokinet Biopharm 1991, 19:537-552.

6. Proost JH, Wierda JM, Meijer DK: An extended pharmacokinetic/pharmacodynamic model describing quantitatively the influence of plasma protein binding, tissue binding, and receptor binding on the potency and time course of action of drugs. Journal of Pharmacokinetics and Biopharmaceutics 1996, 24:45-77.

7. Gibaldi M, Perrier D: Pharmacokinetics. 2nd edition. New York and Basel, Marcel Dekker, Inc.; 1982.

8. Fambrough DM, Drachman DB, Satyamurti S: Neuromuscular junction in myasthenia gravis: decreased acetylcholine receptors. Science 1973, I82:293-295.

9. Pestronk ADDBSSG: Measurement of junctional acetylcholine receptors in myasthenia gravis: Clinical correlates. Muscle \& Nerve 1985, 8:245-25I.

10. Amaki Y, Waud BE, Waud DR: Atracurium-receptor kinetics: simple behavior from a mixture. Anesth Analg 1985, 64:777-780.

II. Fisher DM, Szenohradszky J, Wright PM, Lau M, Brown R, Sharma M: Pharmacodynamic modeling of vecuronium-induced twitch depression. Rapid plasma-effect site equilibration explains faster onset at resistant laryngeal muscles than at the adductor pollicis. Anesthesiology 1997, 86:558-566.

12. Jacquez JA: Compartmental analysis in biology and Medicine. 3rd edition. Dexter, MI, Thompson-Shore, Inc.; 1996.

13. Eccles JC, jaeger JC: The relationship between the mode of operation and the dimensions of the junctional regions at synapses and motor end-organs. Proc R Soc Lond B Biol Sci 1958, 148:38-56.
14. Lentner C: Geigy Scientific Tables. Volume V. 8th edition. West Caldwell, NJ, CIBA-GEIGY Corp.; 1990:222.
Publish with Bio Med Central and every scientist can read your work free of charge

"BioMed Central will be the most significant development for disseminating the results of biomedical research in our lifetime. "

Sir Paul Nurse, Cancer Research UK

Your research papers will be:

- available free of charge to the entire biomedical community

- peer reviewed and published immediately upon acceptance

- cited in PubMed and archived on PubMed Central

- yours - you keep the copyright 